\section{Anatomy and Clinical Relevance of the "Corona Mortis": A Review of the Literature and Current Aspects of Management}

Keywords: Corona mortis; Incidence; Anatomy; Pelvic vessels: Pelvic haemorrhage

\begin{abstract}
Background: The corona mortis is defined as the vascular connections between the obturator and external iliac systems. These small vessels anastomose behind the pubic symphysis between the obturator and external iliac arteries and have been described in classic anatomy texts. This article reviewed the literature in relation to incidence, anatomy and clinical relevance of the corona mortis.
\end{abstract}

Methods: A systematic search was performed using MEDLINE, EMBASE and CINAHL. Both cadaveric and clinical studies were included. The review identified 38 articles published between 1968 and 2015. Studies were analyzed with narrative data synthesis.

Results: There was potential discrepancy between anatomic cadaveric studies and clinical studies in terms of incidence and location. The present study reviewed the relevant literature and provides a comprehensive outline of the anatomy and clinical importance of the corona mortis vessels. We identified reports of both arterial and venous 'corona mortis' with a preponderance for venous vessels.

Conclusions: There is paucity in the report of adverse events in relation to the corona mortis. Nevertheless, special attention needs to be paid to these vascular anastomoses in the context of pelvic trauma, pelvic surgery, lower abdominal wall hernia as well as laparoscopic procedures.

\section{Introduction}

Haemorrhage remains the leading cause of mortality associated with pelvic trauma [1]. The bleeding source can be vascular, osseous or visceral, but most commonly combined venous and osseous bleeding [2]. One potential source of significant bleeding is the presence of accessory or aberrant anastomotic vessels on the superior pubic ramus [3]. These vascular connections are known as the corona mortis, Latin for "crown of death" [4,5]. They occur between the obturator vessels, pubic branches of the obturator vessels and branches from the external iliac vessels [5]. An accessory obturator artery or vein is defined as the presence of at least one extra vessel in addition to the normal counterpart [4], while an aberrant obturator vessel arises from the external iliac or one of its branches [5]. The corona mortis ominous name reflects its significance in pelvic trauma, particularly pubic symphysis and superior pubic ramus fractures (Figure 1). This structure is also at risk during surgical approaches to the pelvis, acetabulum, hernia surgery, and endovascular interventions. This review articles presents the findings on the most relevant literature reporting on the so called 'corona mortis'. The aim of this review was to heighten the awareness of the potential for haemorrhage

\section{Journal of}

Orthopedics \& Rheumatology

\section{Tarek Boutefnouchet ${ }^{1,2^{*}}$, James Bassett ${ }^{2}$ and Sunit Patil ${ }^{1}$}

${ }^{I}$ Trauma \& Orthopaedic Department, University Hospital Coventry and Warwickshire, Clifford Bridge Road, Walsgrave, Coventry, CV2 2DX, UK

${ }^{2}$ College of Medical and Dental Sciences, The University of Birmingham, Edgbaston, Birmingham, B15 2TT, UK

*Address for Correspondence

Tarek Boutefnouchet, MBChB, MRCS, PGCert ME, Specialist Registrar, Trauma \& Orthopaedic Department, University Hospital, Coventry and Warwickshire, Clifford Bridge Road, Walsgrave, Coventry, CV2 2DX, UK, Tel: +44(0)7809677302; E-mail: tboutefnouchet@hotmail.com

Submission: 31 August, 2016

Accepted: 28 September, 2016

Published: 05 October, 2016

Copyright: @ 2016 Boutefnouchet T, et al. This is an open access article distributed under the Creative Commons Attribution License, which permits unrestricted use, distribution, and reproduction in any medium, provided the original work is properly cited.

from the corona mortis among trauma surgeons, general surgeons and interventional radiologists. The authors discuss its anatomy, incidence, classification and present results of their own cadaveric dissections shown in Figures 1 and 2. The present article highlights the importance of the corona mortis in trauma and surgical approaches.

\section{Materials and Methods}

A systematic search of the literature was performed using combined terms related to corona mortis, aberrant obturator vessels, incidence, anatomy, pelvic vessels, and pelvic haemorrhage. The query syntax also used eponyms and generic search terms related to the subject domain and included in the search. Search database used were: MEDLINE', Embase $^{\mathrm{TM}}$, and CINAHL (cumulative index to nursing and allied health literature). Search dates intervals included

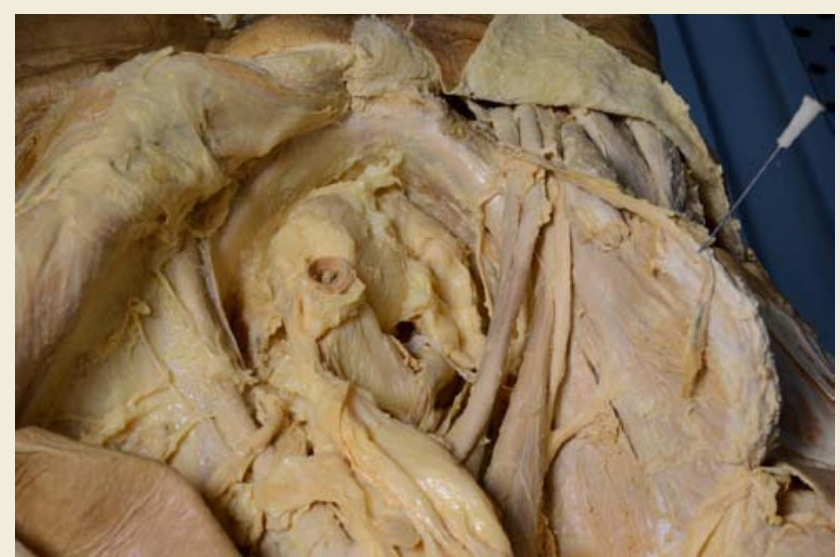

Figure 1: Dissection of embalmed cadaveric specimen. Pelvic dissection with identification of the intra and extra pelvic vasculature and branches forming the corona mortis. External iliac artery and vein (EIA \& EIV), inferior epigastric artery and vein (IEA \& IEV), Common iliac artery and vein (CIA \& CIV). 
Citation: Boutefnouchet T, Bassett J, Patil S. Anatomy and Clinical Relevance of the 'Corona Mortis': A Review of the Literature and Current Aspects of Management. J Orthopedics Rheumatol. 2016; 3(2):5.

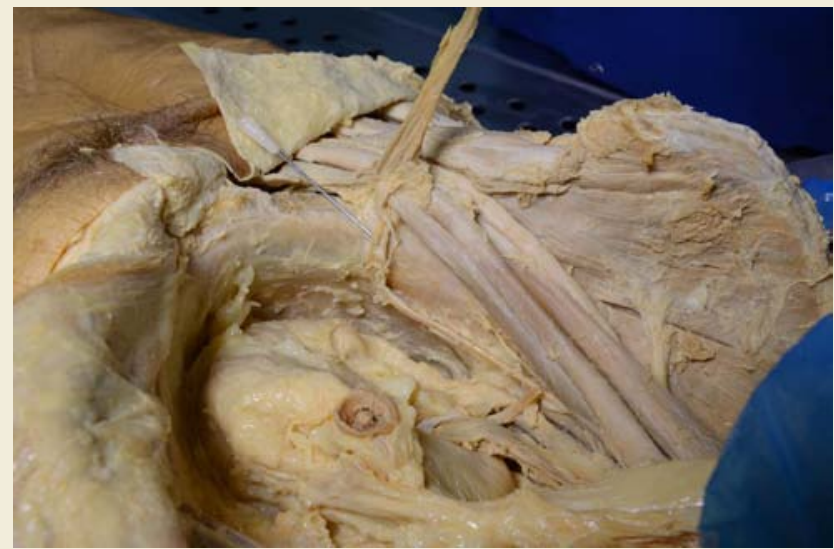

Figure 2: Dissection of embalmed cadaveric specimen. Venous and arterial corona mortis identified connecting the external iliac and obturator vessels (Needle pointing out the corona mortis vessels).

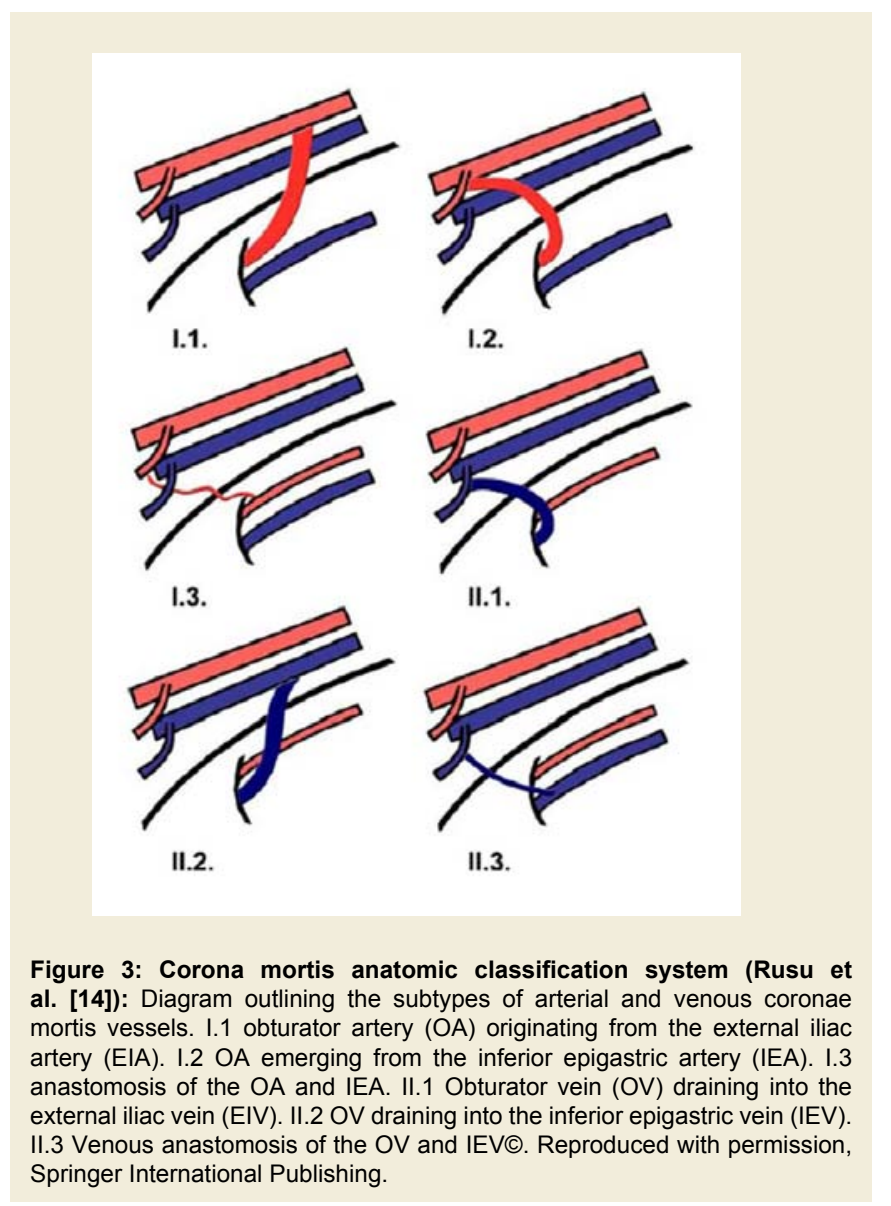

were from their year of inception to the last week of March 2014. PubMed was used as the primary source and search engine for MEDLINE and MeSH (Medical Subject Headings) were used. The same terms were used as search keywords for the other sources. The search yielded 38 articles published from 1968 to January 2015. In addition, a check was carried out on the reference lists for the full text articles generated in order to ensure inclusion of papers not quoted in the electronic database. The articles included in the review were appraised for their pertinence to the anatomy, as well as incidence and clinical relevance of the corona mortis. Cadaveric specimens were dissected for utilisation in teaching and illustration of articles. All procedures performed in studies involving human cadaveric participants were in accordance with the ethical standards of the institutional and national research committee and with the 1964 Helsinki declaration and its later amendments.

\section{Results}

\section{Gross anatomy}

The corona mortis is defined as the vascular connection between the obturator and the external iliac systems [6,7], and may be venous, arterial, or both. This is shown in our own dissected cadaveric specimen on Figures 1 and 2. However, no single pattern predominates, and it can consist of a large branch originating from the inferior epigastric vessel or a network of anastomoses between the pubic branches of the obturator vessels and those of the external iliac vessels. These connections can occur either directly or through the inferior epigastric vessels anastomosing on the upper margin of the obturator foramen $[5,8]$. Renowned anatomists from the $18^{\text {th }}$ and $19^{\text {th }}$ century recognised the significance and frequency of anastomoses between the obturator artery and the inferior epigastric artery [9]. Although classic anatomical texts only describe arterial corona mortis, more recent studies described the presence of venous connections $[10,11]$. The reported incidence varies form $34 \%$ to $60 \%$ and $19 \%$ to $70 \%$ for venous and arterial corona mortis, respectively [10-12]. Several terms have been linked to the corona mortis, such as accessory obturator artery, accessory obturator vein, aberrant obturator artery and anomalous origin of obturator artery [4]. The term corona mortis is used exclusively to describe large anomalous connections, while small calibre anastomoses are believed to be a normal pattern [13]. The average reported diameter according to one series, which looked at both cadaveric, and patients specimen was of $2.6 \mathrm{~mm}$ (range $1.6-3.5 \mathrm{~mm}$ ) [10]. Tornetta and colleagues found vascular channels consistent with a corona mortis in $84 \%$ of 50 fresh cadaveric hemipelvises [12]. Rusu and colleagues classified the corona mortis based on pattern and type of vessels involves. In their classification, there were three types of corona mortis: Type I-arterial; Type II-venous and Type III-a combined arteriovenous vascular network [14]. The arterial anastomoses were sub-classified according to the origin of the obturator artery into: I.1 originating from the external iliac artery, I.2 emerging from the inferior epigastric artery, I.3 anastomosis of the obturator artery and inferior epigastric artery and I.4 Non-anastomosed supra pubic branches of the obturator artery (Figure 3) [14]. The venous connections were further classified into three subtypes: II.1 Obturator vein draining into the external iliac vein, II.2 Obturator vein draining into the inferior epigastric vein and II.3 Multiple venous anastomosis of the obturator vein and inferior epigastric vein. The anatomic classification system proposed by Rusu et al. is outlined further in Figure 3.

\section{Incidence}

The highest incidence reported was $96 \%$ found among 50 cadaveric dissections [8]. Other authors have reported similar incidence and this has been attributed to normal variation rather than abnormal or unusual anastomoses $[15,16]$. The differences in identification of 
Citation: Boutefnouchet T, Bassett J, Patil S. Anatomy and Clinical Relevance of the 'Corona Mortis': A Review of the Literature and Current Aspects of Management. J Orthopedics Rheumatol. 2016; 3(2):5.

ISSN: $2334-2846$

Table 1: Incidence of vascular anastomosis between the obturator and external iliac vessels.

\begin{tabular}{|l|c|c|}
\hline \multicolumn{1}{|c|}{ Study } & N (Specimens) & $\begin{array}{c}\text { Incidence of vascular anastomosis between } \\
\text { obturator and external iliac vessels (\%) }\end{array}$ \\
\hline Darmanis et al. [10] & 80 (cadavres) & 83 \\
Okcu et al. [11] & 150 (cadavres) \\
Lau et al. [18] & 141 (patients) & 61 \\
Karakurt et al. [17] & 98 (cadavres) & 22.2 \\
Berberoglu et al. [8] & 14 (cadavres) & 29 \\
Gilroy et al. [15] & 26 (patients) & 14.2 \\
Tornetta et al. [12] & 105 (cadavres) & 3.3 \\
Namking et al. [16] & 50 (cadavres) & 35.2 \\
Pick et al. [9] & 204 (cadavres) & 34 \\
& 640 (cadavres) & 70.6 \\
\end{tabular}

Table 2: Distance of the corona mortis vessels from the symphysis pubis.

\begin{tabular}{|l|c|c|}
\hline \multicolumn{1}{|c|}{ Study } & N (Specimens) & $\begin{array}{c}\text { Average distance of 'corona mortis' vessels from } \\
\text { the symphysis pubis (mm) }\end{array}$ \\
\hline Darmanis et al. [10] & 80 (cadavres) & Art. 71 \\
Ven. 65 & Art. 64 \\
Okcu et al. [11] & 150 (cadavres) & Ven. 56 \\
Sarikcioglu et al. [4] & & 39.8 \\
Karakurt et al. [17] & 27 (cadavres) & 34 \\
Berberoglu et al. [8] & 98 (cadavres) & 14 (cadavres) \\
Tornetta et al. [12] & 50 (cadavers) & 40.4 \\
\hline
\end{tabular}

Art: Arterial; Ven: Venous

vessels numbers and sizes led to ambiguity about the true incidence of the corona mortis [5]. Sakthivelavan and colleagues reported an incidence of $30 \%$ to $40 \%$ of accessory or aberrant obturator vessels. In another series $84 \%$ of cadaveric dissections had significant vascular connections between the obturator and external iliac vessels [12]. There was no reported gender difference in the incidence. Other cadaveric studies demonstrated that venous tributaries from the internal iliac vessels connecting the inferior epigastric or the external iliac vessels had double the incidence of their arterial counterparts $[15,16]$. The incidence of combined venous-arterial corona mortis was reported in $17.2 \%$ of specimens [16]. Table 1 outlines the reported incidence of vascular anastomoses between the obturator and external iliac vessels.

\section{Location}

The corona mortis vessels are found on the superior margin of the superior pubic ramus [5]. Tornetta et al. reported an average distance of $60 \mathrm{~mm}$ from the symphysis pubis [12]. Okcu and colleagues showed that arterial corona mortis were on average located at $64 \mathrm{~mm}$ from the symphysis pubis (range $45-90 \mathrm{~mm}$ ), whereas venous coronae mortis were located at $56 \mathrm{~mm}$ from the symphysis pubis (range $37-80 \mathrm{~mm}$ ) [11]. The difference in arterial and venous corona mortis location was also reported by Darmanis et al. with an average distance from the symphysis pubis of $71 \mathrm{~mm}$ for arterial vessels and $65 \mathrm{~mm}$ for venous vessels [10]. According to one clinical study, the average distance of the corona mortis form symphysis pubis was $32 \mathrm{~mm}$ in males and 36 $\mathrm{mm}$ in females [17]. The average reported distances of corona mortis vessels from the symphysis pubis are described further in Table 2.

\section{Discussion}

With variation in their location, size and distribution such vascular connections over the superior pubic ramus have a reported high incidence. The corona mortis certainly deserves its name although the incidence of these vessels seems to be overestimated in cadaveric studies in comparison with clinical reports as demonstrated above. The variations seen across cadaveric studies have been attributed to the difficulties in dissecting collapsed cadaveric vessels and the variations in dissecting approaches [11]. Other theories have been postulated by various authors on the reason for the observed variations between cadaveric and clinical studies such as, the demographic and ethnic differences in various study populations and the lack of definition to what truly constitutes a corona mortis [18]. Additionally, in the clinical setting, pelvic trauma tends to induce vessel spasm and retraction into the pelvis making them less readily identifiable [10] or the vessels may have been lacerated by the pubic ramus fracture and haemostasis achieved spontaneously [19]. Finally, cadaveric specimens come from older population with a richer network of collateral circulation due to potential concomitant arterial atherosclerosis and venous thrombosis $[3,4,18]$.

Clinicians involved in the care of pelvic trauma, surgery of the pelvis, hernia surgery and endovascular intervention need to be familiar with the corona mortis. Failure to recognise and take appropriate precautions can result in catastrophic haemorrhage from this rich vascular network. The uncertainty about the true clinical incidence indicates that the corona mortis should not dictate change of surgical practice and operative approaches as long as the surgeon is aware and able to identify and deal with this frequent variation. In contrast with earlier reports more recent studies revealed a higher incidence of venous corona mortis $[14,20,21]$. This we believe has implications with regards to the risk of inadvertent injury to these vessels during surgery and attempts to control bleeding following pelvic trauma using angiographic intervention.

\section{Pelvic trauma}

Half of the mortality associated with pelvic trauma is secondary 
to haemorrhage typically associated with high-energy trauma $[1,22]$. However, life-threatening haemorrhage can occur in seemingly stable low energy fractures of the pubic rami secondary to direct laceration of the corona mortis [23]. Unfortunately, because of the lack of awareness of this structure, the source of bleeding can go unrecognised in that group of patients $[13,20,24]$. CT angiogram (CTA) has been shown to be effective in identifying the corona mortis $[25,26]$. Unfortunately, injury to the corona mortis will result in vascular spasm making it more challenging to identify. The reported sensitivity of CTA identification of the corona mortis in non-pelvic and pelvic fracture patients was $90 \%$ and $63.6 \%$ respectively $[21,24]$. More recently, it has been suggested that angiography is the optimal investigation modality in identifying bleeding corona mortis [20,24]. Additionally, it carries the advantage of being potentially therapeutic if the bleeding point can be identified and embolised. It is also important to note that in order to effectively identify the corona mortis with angiography, selective cannulation of the inferior epigastric vessel is crucial. The importance of angiographic embolization in managing haemorrhage in pelvic trauma has now become well established [22,27]. Delayed angiography due to prolonged resuscitation, laparotomy or further diagnostic tests is associated with higher mortality [22,27], mandating a decision for early angiography where suspicion is high, particularly if a contrast trauma CT has identified extravasation of contrast near fractured pubic rami.

\section{Surgical approaches}

Corona mortis vessels are at risk in surgical approaches near the pubic rami, and are thus of potential concern to the orthopaedic surgeon, urologist, gynaecologist and general surgeons. Bleeding from the corona mortis vessels if damaged can be difficult to control as vessels retract into the pelvis increasing the risks of morbidity and mortality associated with surgery on the anterior aspect of the pelvic ring [4]. These vessels are especially at risk with the ilioinguinal approach to the pelvis and acetabulum, and pelvic osteotomies carried out through this approach [28]. This extensile surgical approach offers access from the sacroiliac joint to the pubic symphysis without causing any insult to the hip joint, and consequently gained significant popularity. In a study where dissection of cadaveric hemipelvises was carried out using the ilioinguinal approach, vascular anastomoses consistent with a description of the corona mortis were found in $61 \%$ of specimens [11]. Letournel reported identifying corona mortis vessels in $10 \%-15 \%$ of his cases recommending awareness and early recognition of these vessels in order to reduce the risk of intractable haemorrhage [19]. During the ilioinguinal approach, Letournel recommended inserting a finger during dissection below the external iliac vessels from lateral to medial and application of a rubber sling around those vessels branching from the iliac vessels and origin of a potential corona mortis. The posterior-superior area of the superior pubic ramus should be carefully palpated for any large or pulsating vessels. In addition, gentle retraction of the external iliac vessels with the sling, allows identification of any possible significant retropubic vessels or 'corona mortis' $[10,19]$. Several authors reported their experience in anterior approaches using the ilioinguinal and the modified intrapelvic Stoppa approaches $[10,29,30]$. These approaches were used for operative fixation of fractures involving the pelvic ring and displaced fractures of the acetabulum, especially of the anterior wall and anterior column. Darmanis et al. reported their experience with a high volume series exceeding 400 cases, the authors encountered corona mortis vessels that required ligation in five patients. In an additional case, the corona mortis was accidentally cut resulting in torrential haemorrhage and requiring massive blood transfusion [10]. Similarly Teague and colleagues reported their findings during ilioinguinal surgical approaches for fixation of displaced acetabular fractures in 38 consecutive cases [28]. In this study, vessels corresponding to the corona mortis topography were identified in $37 \%$ of cases, these vessels were ligated as part of the approach and no adverse events were reported.

The corona mortis has equally significant implications in surgical approaches to hernia repair especially femoral and obturator hernias and more recently with endoscopic hernia surgery [8,18]. Close proximity to the lacunar ligament means these vessels are especially at risk during the release of a femoral canal hernia. Studies using preperitoneal endoscopic inguinal hernia repair demonstrate findings of corona mortis vessels in $40 \%$ of cases [18]. Others reported venous connections in $96 \%$ of cases [8]. The majority of these anastomoses were lateral to the hernial sac, oriented vertically and were particularly prone to injury during dissection [18]. Venous bleeding can be initially inconspicuous and results in large haematoma formation if undetected [17].

Surgical awareness of the corona mortis is important in both trauma patients and operative surgical approaches to the pelvis and groin region. There have been discrepancies in the reported incidence and location of this vascular network. Therefore more cadaveric and clinical imaging studies are required in order to identify the true extent and variance of the corona mortis. With the advent of threedimensional imaging modalities and ease of access to Trauma CT in major Trauma Centre from the outset of patient's management, the authors recommend the potential need for identifying the presence of corona mortis vessels which can help in dealing with these vessels intra-operatively. Further studies can help elucidate the impact of such practice on intra-operative blood loss.

\section{References}

1. Giannoudis PV, Grotz MR, Tzioupis C, Dinopoulos H, Wells GE, et al. (2007) Prevalence of pelvic fractures, associated injuries, and mortality: the United Kingdom perspective. J Trauma 63: 875-883.

2. Gustavo Parreira J, Coimbra R, Rasslan S, Oliveira A, Fregoneze M, et al. (2000) The role of associated injuries on outcome of blunt trauma patients sustaining pelvic fractures. Injury 31: 677-682.

3. Daeubler B, Anderson SE, Leunig M, Triller J (2003) Hemorrhage secondary to pelvic fracture: coil embolization of an aberrant obturator artery. J Endovasc Ther 10: 676-680.

4. Sarikcioglu L, Sindel M, Akyildiz F, Gur S (2003) Anastomotic vessels in the retropubic region: corona mortis. Folia Morphol (Warsz) 62: 179-182.

5. Sakthivelavan S, Sendiladibban SD, Aristotle S, Sivanandan AV (2010) Corona mortis: a case report with surgical implications. Int J Anat Var 3: 103105.

6. Moore KL, Dalley AF, Agur AM (2010) Clinically oriented anatomy. $6^{\text {th }}$ edn Lippincott Williams and Wilkins, Baltimore, J Anat 215: 474.

7. Tank PW (2008) Grant's dissector. $14^{\text {th }}$ edn, Lippincott Williams \& Wilkins, pp. 288.

8. Berberoglu M, Uz A, Ozmen MM, Bozkurt MC, Erkuran C, et al. (2001) Corona mortis: an anatomic study in seven cadavers and an endoscopic study in 28 patients. Surg Endosc 15: 72-75. 
Citation: Boutefnouchet T, Bassett J, Patil S. Anatomy and Clinical Relevance of the 'Corona Mortis': A Review of the Literature and Current Aspects of Management. J Orthopedics Rheumatol. 2016; 3(2):5.

ISSN: $2334-2846$

9. Pick JW, Anson BJ, Ashley FL (1942) The origin of the obturator artery: a study of 640 body-halves. Am J Anat 70: 317-343.

10. Darmanis S, Lewis A, Mansoor A, Bircher M (2007) Corona mortis: an anatomical study with clinical implications in approaches to the pelvis and acetabulum. Clin Anat 20: 433-439.

11. Okcu G, Erkan S, Yercan HS, Ozic U (2004) The incidence and location of corona mortis: a study on 75 cadavers. Acta Orthop Scand 75: 53-55.

12. Tornetta P 3rd, Hochwald N, Levine R (1996) Corona mortis. Incidence and location. Clin Orthop Relat Res 97-101.

13. Marsman JW, Schilstra SH, van Leeuwen H (1984) Angiography and embolization of the corona mortis (aberrant obturator artery). A source of persistent pelvic bleeding. Rofo 141: 708-710.

14. Rusu MC, Cergan R, Motoc AG, Folescu R, Pop E (2010) Anatomical considerations on the corona mortis. Surg Radiol Anat 32: 17-24.

15. Gilroy AM, Hermey DC, DiBenedetto LM, Marks SC Jr, Page DW, et al. (1997) Variability of the obturator vessels. Clin Anat 10: 328-332.

16. Namking $M$, Woraputtaporn W, Buranarugs $M$, Kerdkunchorn $M$ Chaijarakhumarak W (2007) Variations in origin of the obturator artery and corona mortis. Siriraj Med J 59: 12-15.

17. Karakurt L, Karaca I, Yilmaz E, Burma O, Serin E (2002) Corona mortis: incidence and location. Arch Orthop Trauma Surg 122: 163-164.

18. Lau H, Lee $F$ (2003) A prospective endoscopic study of retropubic vascular anatomy in 121 patients undergoing endoscopic extraperitoneal inguinal hernioplasty. Surg Endosc 17: 1376-1379.

19. Letournel E (1993) The treatment of acetabular fractures through the ilioinguinal approach. Clin Orthop Relat Res 62-76.

20. Garrido-Gomez J, Pena-Rodriguez C, Martin-Noguerol T, Hernandez-Cortes
P (2012) Corona mortis artery avulsion due to a stable pubic ramus fracture. Orthopedics 35: e80-e82.

21. Hallinan JT, Tan CH, Pua U (2014) Emergency computed tomography for acute pelvic trauma: where is the bleeder? Clin Radiol 69: 529-537.

22. Tosounidis TI, Giannoudis PV (2013) Pelvic fractures presenting with haemodynamic instability: treatment options and outcomes. Surgeon 11 344-351.

23. Dondelinger RF, Trotteur G, Ghaye B, Szapiro D (2002) Traumatic injuries: radiological hemostatic intervention at admission. Eur Radiol 12: 979-993.

24. Pua U, Teo LT (2012) Prospective diagnosis of corona mortis hemorrhage in pelvic trauma. J Vasc Interv Radiol 23: 571-573.

25. Perez JV, Hughes TM, Bowers K (1998) Angiographic embolisation in pelvic fracture. Injury 29: 187-191.

26. Agolini SF, Shah K, Jaffe J, Newcomb J, Rhodes M, et al. (1997) Arterial embolization is a rapid and effective technique for controlling pelvic fracture hemorrhage. J Trauma 43: 395-399.

27. Yoon W, Kim JK, Jeong YY, Seo JJ, Park JG, et al. (2004) Pelvic arteria hemorrhage in patients with pelvic fractures: detection with contrastenhanced CT. Radiographics 24: 1591-1605.

28. Teague DC, Graney DO, Routt ML Jr (1996) Retropubic vascular hazards of the ilioinguinal exposure: a cadaveric and clinical study. J Orthop Trauma 10: 156-159.

29. Cole JD, Bolhofner BR (1994) Acetabular fracture fixation via a modified Stoppa limited intrapelvic approach. Description of operative technique and preliminary treatment results. Clin Orthop Relat Res 112-123.

30. Sagi HC, Afsari A, Dziadosz D (2010) The anterior intra-pelvic (modified rives-stoppa) approach for fixation of acetabular fractures. J Orthop Trauma 24: $263-270$

\section{Acknowledgements}

The authors of this article formally acknowledge the enormous contribution made by those who donated their bodies for education and research. We recognise their donation as an unparalleled gift to education and research without which the advancement of our field will be severely hampered. 